Article

\title{
Expression of Terpenoid Biosynthetic Genes and Accumulation of Chemical Constituents in Valeriana fauriei
}

\author{
Yun Ji Park ${ }^{1}$, Mariadhas Valan Arasu ${ }^{2}$, Naif Abdullah Al-Dhabi ${ }^{2}$, Soon Sung Lim ${ }^{3}$, \\ Yeon Bok Kim ${ }^{4}$, Sang Won Lee ${ }^{4}$ and Sang Un Park ${ }^{1, *}$ \\ 1 Department of Crop Science, Chungnam National University, 99 Daehak-ro, Yuseong-gu, Daejeon 34134, \\ Korea; yunji0825@hanmail.net \\ 2 Department of Botany and Microbiology, Addiriyah Chair for Environmental Studies, College of Science, \\ King Saud University, P. O. Box 2455, Riyadh 11451, Saudi Arabia; mvalanarasu@gmail.com (M.V.A.); \\ naldhabi@ksu.edu.sa (N.A.A.-D.) \\ 3 Department of Food and Nutrition and Institute of Natural Medicine, Hallym University, \\ Chuncheon 200-702, Korea; limss@hallym.ac.kr \\ 4 Department of Herbal Crop Research, National Institute of Horticultural and Herbal Science (NIHHS), \\ Rural Development Administration (RDA), Bisanro 92, Eumseong, Chungbuk 369-873, Korea; \\ yeondarabok@korea.kr (Y.B.K.); swlee1004@korea.kr (S.W.L.) \\ * Correspondence: supark@cnu.ac.kr; Tel.: +82-42-821-6730; Fax: +82-42-822-2631
}

Academic Editor: Tobias A. M. Gulder

Received: 2 April 2016; Accepted: 20 May 2016; Published: 27 May 2016

\begin{abstract}
Valeriana fauriei (V. fauriei), which emits a characteristic and unpleasant odor, is important in traditional medicine. In this study, the expression of terpenoid biosynthetic genes was investigated in different organs that were also screened for volatile compounds including valerenic acid and its derivatives. Specific expression patterns from different parts of $V$. fauriei were observed using quantitative real-time PCR (qRT-PCR). The highest transcript levels of biosynthetic genes involved in mevalonic acid (MVA) and methylerythritol phosphate (MEP) production were found in the stem. Although the amounts of volatile compounds were varied by organ, most of the volatile terpenoids were accumulated in the root. Gas chromatography mass spectrometry (GC-MS) analysis identified 128 volatile compounds, which represented $65.33 \%$ to $95.66 \%$ of total volatiles. Certain compounds were only found in specific organs. For example, isovalerenic acid and valerenic acid and its derivatives were restricted to the root. Organs with high transcript levels did not necessarily have high levels of the corresponding chemical constituents. According to these results, we hypothesize that translocation may occur between different organs in $V$. fauriei.
\end{abstract}

Keywords: gene expression; terpenoid; valerenic acid; Valeriana fauriei; volatile compounds

\section{Introduction}

Plants have complex mixtures of volatile lipophilic compounds with low molecular weight and high vapor pressure, derived from both primary and secondary metabolisms [1]. More than 1700 volatile compounds have been characterized from 90 plant families [2]. Plants release these compounds for general or specialized functions in both floral and vegetative tissues [3]. These compounds protect against herbivores, pathogens, and parasites, attract pollinators and seed dispersers, and provide plant-plant signaling [4]. Additionally, volatile compounds emitted from plants can seal wounds [5]. For millennia, humans have used floral scents from many aromatic plants, intended to attract pollinators, as sources of flavorings, preservatives, and herbal remedies [6]. Researches have documented antimicrobial, anti-inflammatory, bronchodilatory, expectorant, anticonvulsant, 
cholagogic, analgesic, and spasmolytic effects of volatile compounds [7]. Volatile compounds determine a plant's chemotype and can influence its ecological relevance by shaping interactions with pollinators and herbivores [8]. Diverse volatile compounds are synthesized by different biochemical pathways in plants. There are lipoxygenase pathways including oxylipins, green leaf volatiles, isoprene and other terpenoids, some carotenoid derivatives, indoles, and phenolics, such as methyl salicylate [9].

Terpenoids, which constitute the largest class of volatiles from plants, are synthesized from isoprenoid pathways (Figure 1) [1]. There are three basic phases of volatile terpenoid biosynthesis [10]. First, $\mathrm{C}_{5}$-isoprene units are formed from isopentenyl pyrophosphate (IPP) and its isomer dimethylallyl pyrophosphate (DMAPP) via two compartmentally separated pathways, the mevalonic acid (MVA) pathway in the cytoplasm and the methylerythritol phosphate (MEP) pathway in the chloroplast. These precursors are then catalyzed by short-chain prenyltransferase to form prenyl diphosphates, including geranyl diphosphate (GPP), farnesyl diphosphate (FPP), and geranylgeranyl pyrophosphate (GGPP) [11,12]. Finally, many terpene synthases (TPS) synthesize prenyl diphosphates and build primary representatives of each type of terpenoid skeleton. Many volatile terpenoids are directly catalyzed by TPS, but others are modified by oxidation, dehydrogenation, acylation, and other types of reactions [10]. The MVA pathway is thought to provide three $C_{5}$ units for volatile sesquiterpenes $\left(C_{15}\right)$, while the MEP pathway gives rise to volatile hemiterpenes $\left(C_{5}\right)$, monoterpenes $\left(C_{10}\right)$, and diterpenes $\left(C_{20}\right)$ [13]. However, metabolic cross talk has been demonstrated, regulated by exchange of isoprenes like IPP between cytosolic and chloroplastic pathways [14].

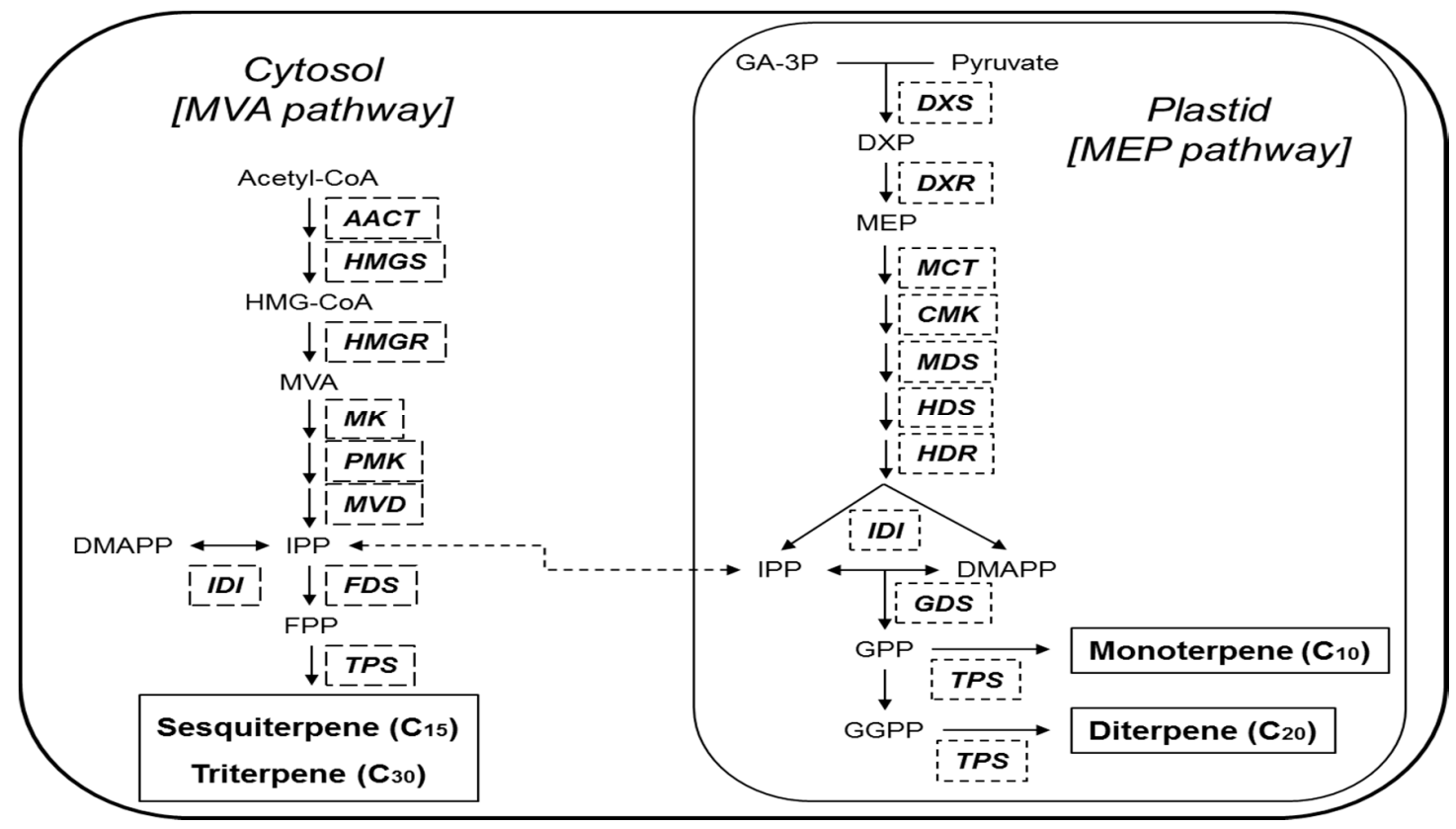

Figure 1. Volatile terpenoid biosynthetic pathways in plants. AACT, Acetoacetyl-CoA thiolase; $C M K$, 4-(cytidine 5'-diphosphate)-2-C-methyl-D-erythritol kinase; DMAPP, dimethylallyl diphosphate; DXP, 1-deoxy-D-xylulose 5-phosphate; DXR, DXP reductoisomerase; DXS, DXP synthase; FDS, farnesyl diphosphate synthase; FPP, farnesyl diphosphate; GA-3P, glyceraldehyde-3-phosphate; GDS, geranyl diphosphate synthase; GGPP, geranylgeranyl diphosphate; GPP, geranyl diphosphate; HDR, (E)-4-hydroxy-3-methylbut 2-enyl diphosphate reductase; HDS, (E)-4-hydroxy-3-methylbut-2-enyl diphosphate synthase; HMG-CoA, 3-hydroxy-3-methylglutaryl-CoA; HMGR, HMG-CoA reductase; HMGS, HMG-CoA synthase; IDI, isopentenyl diphosphate isomerase; IPP, isopentenyl diphosphate; $M C T$, 2-C-methyl-D-erythritol 4-phosphate cytidylyltransferase; MDS, 2-C-methyl-D-erythritol 2,4-cyclodiphosphate synthase; MEP, 2-C-methyl-D-erythritol 4-phosphate; $M K$, mevalonate kinase; MVA, mevalonate; $M V D$, mevalonate diphosphate decarboxylase; $P M K$, phosphomevalonate kinase; TPS, terpene synthases. 
The genus Valeriana L. belongs to Caprifoliaceae (honeysuckle family), and includes approximately 250 perennial herbaceous species with malodorous root stalks [15], and grows in temperate areas around the world [16]. These species have been used as mild sedatives and tranquilizers in traditional medicine in various cultures since ancient times [17]. The rhizomes and roots are thought to induce sedation, promote sleep, and relieve depression and anxiety. Species of Valeriana are also used in the food, beverage, and cosmetic industries because of their unique flavor [18]. Many studies have demonstrated that extracts from Valeriana plants include terpenoids, iridoids, flavonoids, and alkaloids [16]. Valerenic acid and its derivatives accumulate in the roots and rhizomes in significant quantities [19]. Chemical constituents of Valeriana vary by species and with seasonal variation and ecological factors [16,17].

We profiled overall volatile compounds, including valerenic acid and its derivatives, from different tissues of Valeriana fauriei ( $V$. fauriei) grown in South Korea. In addition, we characterized the expression patterns of terpenoid biosynthetic genes and compared them with patterns of volatile compound accumulation.

\section{Results}

\subsection{Transcript Levels of Terpenoid Biosynthetic Genes}

We determined differential organ-specific expression patterns of terpenoid biosynthetic genes in $V$. fauriei using qRT-PCR (quantitative real time polymerase chain reaction). The results are shown in Figures 2 and 3. The height of each bar and the error bar indicate the mean and standard deviation based on three independent measurements. The Y-axis represents transcript levels normalized to $18 S$ expression. Most genes related to the MVA pathway showed the highest expression levels in the stem, except for VfHMGS and VfHMGR. The expression patterns of VfAACT, VfMK, VfPMK, $V F M V D$, and VfFDS were fairly similar, with the highest levels occurring in the stem, followed by the root and the flower or the leaf. Interestingly, in roots, VfAACT and VfFDS, which are involved in the first and last steps of the MVA pathway, were expressed at similar levels to the stem. Among MVA biosynthetic genes, the expression of VfAACT, VfMK and VfMVD was high relative to 18S, while expression of VfFDS was the lowest relative to $18 S$. VIIDI, which produces the enzyme that catalyzes the isomerization of IPP to DMAPP in both the MVA and MEP pathways, was expressed most abundantly in the stem, followed by the flower, root, and leaf. According to analyses of MEP biosynthetic gene expression levels, most genes, including $V f C M K, V f M D S, V f H D R$, and $V f G D S$, had the highest expression levels in the stem, whereas VfDXR and VfHDS were highly expressed in the leaf. $V F M C T$ had similar expression levels in all organs of $V$. fauriei. The lowest expression levels of $V f D X R$, $V f M D S, V f H D S$, and $V f H D R$ were displayed in the root. In contrast, VfDXS transcripts, which are involved in the start of the MEP biosynthetic pathway, had their highest level in the root. Expression levels of terpenoid backbone biosynthetic genes, which lead to IPP and DMAPP production, were primarily high in stem of $V$. fauriei.

\subsection{Analysis of Volatile Constituents}

In total, 128 volatile compounds were separated and identified based on comparison of the mass spectra with the National Institute of Standards and Technology (NIST, Gaithersburg, MD, USA) atomic spectra database, Wiley Registry of Mass Spectral Data, and the related literature (Table 1) [20]. The total yield of the volatile compounds was indicated by the highest amount in the root (95.66\%), followed by stem $(84.05 \%)$, leaf (74.91\%), and flower (65.33\%). We identified 18 monoterpenes, 34 sesquiterpenes, and 80 other volatile compounds from $V$. fauriei. Monoterpenes, namely borneol (11, 22.96\%), bornyl acetate $(13,19.92 \%)$, and camphene $(1,3.33 \%)$ were found in high amounts in the roots. Interestingly, $\beta$-terpinene (3), myrcene (4), $p$-cymene (5), and borneol (11) were detected only in the root. The leaf extract was characterized by a high content of endo-borneol $(\mathbf{1 0}, 11.48 \%)$ and $\gamma$-terpinene $(\mathbf{7}, 3.23 \%)$. Sesquiterpenes accumulated primarily in the stem $(21.55 \%)$ and roots $(20.66 \%)$. The stem contained the highest amount of the sesquiterpene caryophyllene oxide (34, 17.45\%). Seven sesquiterpenes $(\mathbf{2 0}-\mathbf{2 2}, \mathbf{2 4}-\mathbf{2 6}$, and 50) were root-specific, while four others $(\mathbf{3 3}, \mathbf{3 7}, \mathbf{3 9}$, and 51) were flower-specific. 
Isovalerenic acid (53, 3.33\%), which produces the strong smell of Valeriana species, was found only in the root of $V$. fauriei.

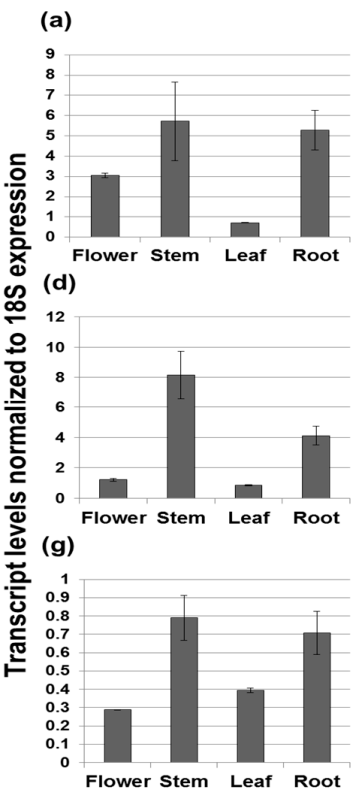

(b)

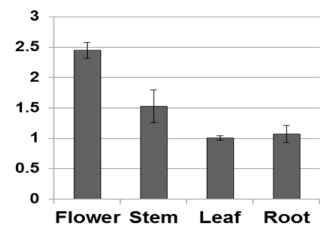

(e)
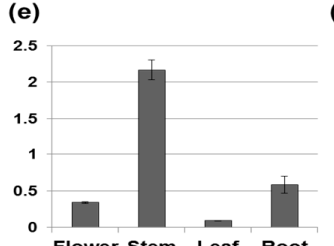

(h)

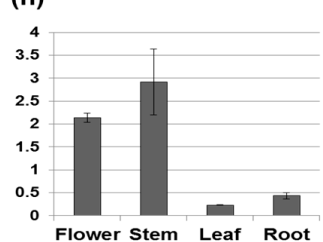

(c)

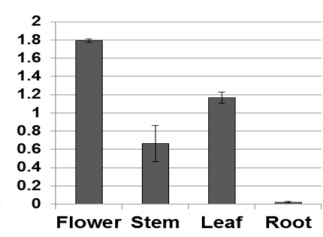

(f)

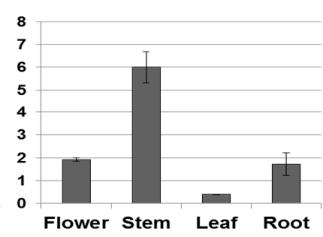

Figure 2. Transcript levels of MVA biosynthetic genes in different organs of $V$. fauriei $(V f)$. Genes encode the following enzymes: (a) VfAACT, acetoacetyl-CoA thiolase; (b) VfHMGS, 3-hydroxy-3methylglutaryl-CoA synthase; (c) VfHMGR, 3-hydroxy-3-methylglutaryl-CoA reductase; (d) VfMK, mevalonate kinase; (e) VfPMK, phosphomevalonate kinase; (f) VfMVD, mevalonate diphosphate decarboxylase; (g) VfFDS, farnesyl diphosphate synthase; and (h) VfIDI, isopentenyl diphosphate isomerase.

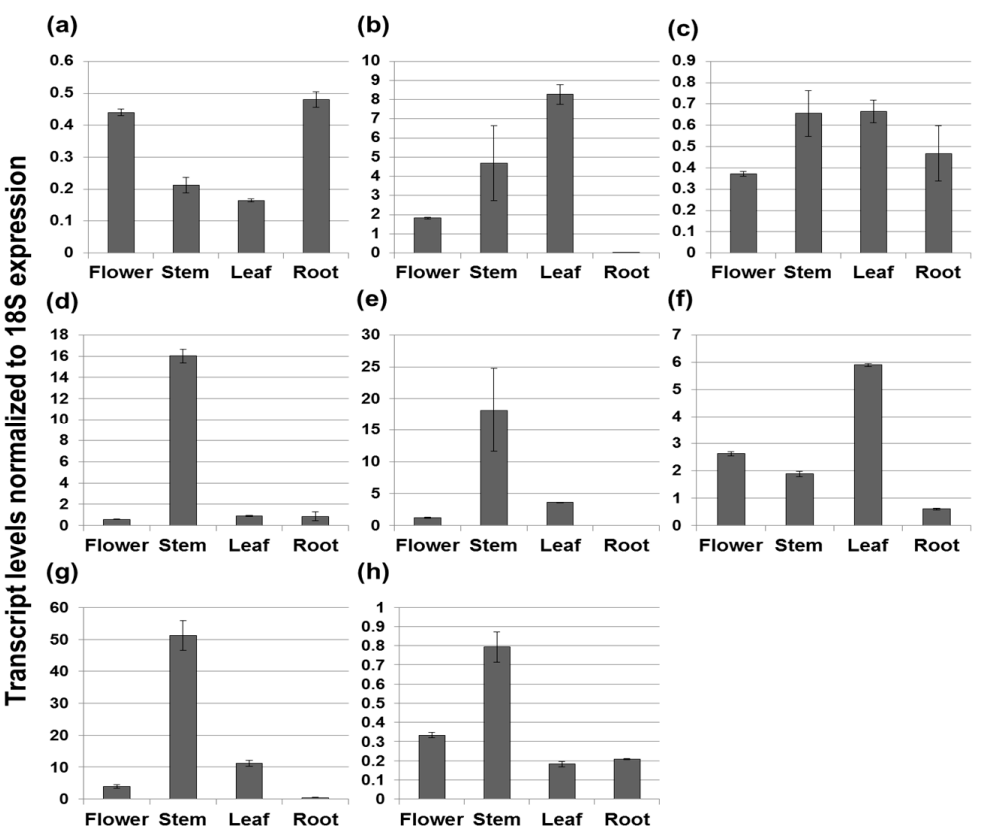

Figure 3. Transcript levels of MEP biosynthetic genes in different organs from $V$. fauriei $(V f)$. Genes encode the following enzymes: (a) VfDXS, 1-deoxy-D-xylulose 5-phosphate synthase; (b) VfDXR, 1-deoxy-D-xylulose 5-phosphate reductoisomerase; (c) VfMCT, 2-C-methyl-D-erythritol 4-phosphate cytidylyltransferase; (d) VfCMK, 4-(cytidine 5'-diphosphate)-2-C-methyl-D-erythritol kinase; (e) VfMDS, 2-C-methyl-D-erythritol 2,4-cyclodiphosphate synthase; (f) VfHDS, (E)-4-hydroxy-3-methylbut-2-enyl diphosphate synthase; (g) VfHDR, (E)-4-hydroxy-3-methylbut 2-enyl diphosphate reductase; and (h) VfGDS, geranyl diphosphate synthase. 
Table 1. Composition of volatile compounds in different organs from $V$. fauriei.

\begin{tabular}{|c|c|c|c|c|c|c|}
\hline \multirow{2}{*}{ No. } & \multirow{2}{*}{ Volatile Compounds } & \multirow{2}{*}{$\mathbf{R I}^{*}$} & \multicolumn{4}{|c|}{ Relative Peak Area (\%) } \\
\hline & & & Flower & Stem & Leaf & Root \\
\hline 1 & $n$-Hexanal & 1.43 & 0 & 0 & 0 & $0.162 \pm 0.004$ \\
\hline 2 & Isovaleric acid & 11.96 & 0 & 0 & 0 & $3.338 \pm 0.082$ \\
\hline 3 & Camphene & 12.91 & 0 & 0 & 0 & $3.338 \pm 0.082$ \\
\hline 4 & $\beta$-Pinene & 13.64 & 0 & $1.244 \pm 0.103$ & 0 & $0.173 \pm 0.0041$ \\
\hline 5 & $\beta$-Terpinene & 14.51 & 0 & 0 & 0 & $0.558 \pm 0.014$ \\
\hline 6 & Myrcene & 15.44 & 0 & 0 & 0 & $0.465 \pm 0.011$ \\
\hline 7 & p-Cymene & 17.22 & 0 & 0 & 0 & $0.085 \pm 0.002$ \\
\hline 8 & $\beta$-Phellandrene & 17.79 & $1.277 \pm 0.111$ & 0 & 0 & $0.803 \pm 0.020$ \\
\hline 9 & $\gamma$-Terpinene & 19.09 & $0.132 \pm 0.011$ & 0 & $3.231 \pm 0.262$ & $0.052 \pm 0.001$ \\
\hline 10 & Terpinolene & 21.35 & 0 & $0.901 \pm 0.074$ & 0 & $0.041 \pm 0.001$ \\
\hline 11 & Ethyl 3-Hydroxymandelate & 21.54 & $0.197 \pm 0.017$ & $0.699 \pm 0.058$ & $5.331 \pm 0.433$ & 0 \\
\hline 12 & D-Limonene & 22.90 & $0.282 \pm 0.024$ & 0 & $\overline{0}$ & $0.082 \pm 0.002$ \\
\hline 13 & 1,3-Bis-( $p$-carbamoylmethylphenoxy)-2-propanol & 23.18 & $0.512 \pm 0.044$ & 0 & 0 & 0 \\
\hline 14 & endo-Borneol & 23.60 & 0 & $0.125 \pm 0.010$ & $11.482 \pm 0.932$ & $2.841 \pm 0.069$ \\
\hline 15 & 1-(4-methoxyphenyl)imidazzoline-2-thione & 24.22 & $0.564 \pm 0.049$ & $0.211 \pm 0.017$ & 0 & 0 \\
\hline 16 & 2,2-Dimethyl-3-heptanone & 24.41 & $\overline{0}$ & 0 & $0.561 \pm 0.046$ & 0 \\
\hline 17 & 1,2,4-Trizol-3-amene & 24.87 & $0.179 \pm 0.016$ & 0 & 0 & 0 \\
\hline 18 & Borneol & 25.11 & 0 & 0 & 0 & $22.96 \pm 0.561$ \\
\hline 19 & Benzoic acid & 25.35 & 0 & $0.966 \pm 0.080$ & 0 & $1.522 \pm 0.037$ \\
\hline 20 & Benzothialzole & 25.87 & $2.725 \pm 0.237$ & 0 & 0 & 0 \\
\hline 21 & Eudesmol & 25.95 & $\overline{0}$ & $0.354 \pm 0.029$ & 0 & $0.077 \pm 0.002$ \\
\hline 22 & 2-Isopropyl-5-methyl anisole & 26.47 & $0.987 \pm 0.086$ & $0.497 \pm 0.041$ & 0 & $\overline{0}$ \\
\hline 23 & Myrtenyl acetate & 26.73 & $\overline{0}$ & $\overline{0}$ & $2.226 \pm 0.181$ & $1.729 \pm 0.042$ \\
\hline 24 & 2-Hexanoylfuran & 27.27 & $0.610 \pm 0.053$ & 0 & $0.556 \pm 0.045$ & $0.132 \pm 0.003$ \\
\hline 25 & Perillaldehyde & 27.57 & $0.834 \pm 0.072$ & 0 & 0 & $1.071 \pm 0.026$ \\
\hline 26 & 9-oxo-(2,6-dimethylpehenyl)amide 9- $\mathrm{H}$-Fluorene-4-carboxylic acid & 27.94 & $1.067 \pm 0.093$ & $0.241 \pm 0.020$ & $21.436 \pm 1.741$ & $0.581 \pm 0.014$ \\
\hline 27 & Acetic acid & 28.31 & $0.612 \pm 0.053$ & $\overline{0}$ & $0.353 \pm 0.029$ & $0.86 \pm 0.021$ \\
\hline 28 & $\alpha$-Gurjunene & 29.74 & $0.640 \pm 0.056$ & 0 & $\overline{0}$ & $0.133 \pm 0.003$ \\
\hline 29 & Bornyl acetate & 30.51 & $0.132 \pm 0.011$ & 0 & 0 & $19.923 \pm 0.487$ \\
\hline 30 & 9H-Fluorene-4-carboxylic acid & 30.77 & $0.266 \pm 0.023$ & $0.306 \pm 0.025$ & $0.253 \pm 0.021$ & $0.087 \pm 0.002$ \\
\hline 31 & $\alpha$-Elemene & 30.88 & 0 & 0 & 0 & $0.038 \pm 0.001$ \\
\hline 32 & 1-Trifluoromethyl-4-(2-emthoxylbenzyloxy)-3-nitro-benzene & 31.06 & $0.298 \pm 0.026$ & $0.078 \pm 0.006$ & 0 & $0.029 \pm 0.001$ \\
\hline 33 & $\mathrm{~N}$-(Cyclohexanecarbonyl)-1-proline isobutyl ester & 31.35 & $0.133 \pm 0.012$ & $\overline{0}$ & 0 & $0.022 \pm 0.001$ \\
\hline 34 & Bromopropylate & 31.97 & $0.461 \pm 0.040$ & $0.367 \pm 0.030$ & 0 & $0.318 \pm 0.008$ \\
\hline 35 & Methadone $N$-oxide & 32.50 & $0.286 \pm 0.025$ & 0 & $2.524 \pm 0.205$ & $0.509 \pm 0.012$ \\
\hline
\end{tabular}


Table 1. Cont.

\begin{tabular}{|c|c|c|c|c|c|c|}
\hline \multirow{2}{*}{ No. } & \multirow{2}{*}{ Volatile Compounds } & \multirow{2}{*}{$\mathbf{R I}$ * } & \multicolumn{4}{|c|}{ Relative Peak Area (\%) } \\
\hline & & & Flower & Stem & Leaf & Root \\
\hline 36 & Quinoline & 33.01 & $0.191 \pm 0.017$ & $0.174 \pm 0.014$ & $1.510 \pm 0.123$ & $1.35 \pm 0.033$ \\
\hline 37 & 2-Chloro-6-methyl-pyridine & 33.40 & $0.190 \pm 0.016$ & $0.226 \pm 0.019$ & $0.456 \pm 0.037$ & $0.065 \pm 0.002$ \\
\hline 38 & Pentadecane & 33.51 & $0.170 \pm 0.015$ & $\overline{0}$ & $\overline{0}$ & $0.095 \pm 0.002$ \\
\hline 39 & 4,6-bis(1-Methylethyl)-trans-1,3-dioxane & 34.00 & 0 & $0.074 \pm 0.006$ & $0.751 \pm 0.061$ & $0.065 \pm 0.002$ \\
\hline 40 & $o$-Choloroaniline & 34.12 & 0 & $0.955 \pm 0.079$ & $1.886 \pm 0.153$ & $\overline{0}$ \\
\hline 41 & $\alpha$-Caryophyllene alcohol & 34.72 & 0 & 0 & 0 & $1.272 \pm 0.031$ \\
\hline 42 & Isolonhifolan-8-ol & 34.81 & 0.8410 .073 & 0 & $0.424 \pm 0.034$ & $0.209 \pm 0.005$ \\
\hline 43 & $\gamma$-Elemene & 34.93 & 0 & 0 & 0 & $0.357 \pm 0.009$ \\
\hline 44 & Caryophyllene & 35.22 & $0.680 \pm 0.059$ & 0 & $0.205 \pm 0.017$ & $0.134 \pm 0.003$ \\
\hline 45 & 5-(Phenylmethyl)-2-thioxo-4-imidazolidinone & 35.37 & 0 & $2.235 \pm 0.0185$ & $0.434 \pm 0.035$ & $\overline{0}$ \\
\hline 46 & $\alpha$-Acorenol & 36.04 & $0.284 \pm 0.025$ & 0 & 0 & $4.025 \pm 0.098$ \\
\hline 47 & cis- $\beta$-Farnesene & 36.18 & $\overline{0}$ & 0 & 0 & $0.041 \pm 0.001$ \\
\hline 48 & Humulene & 36.28 & 0 & 0 & 0 & $0.085 \pm 0.002$ \\
\hline 49 & Aromadendrene & 36.56 & 0 & 0 & 0 & $0.741 \pm 0.018$ \\
\hline 50 & Alloaromandendrene & 36.71 & $1.162 \pm 0.101$ & 0 & 0 & $0.066 \pm 0.002$ \\
\hline 51 & Ylangene & 36.92 & $\overline{0}$ & $0.299 \pm 0.025$ & $0.779 \pm 0.063$ & $2.878 \pm 0.070$ \\
\hline 52 & 1-(1,5-Dimethyl-4-hexenyl)-4-methyl-benzene & 37.13 & $1.181 \pm 0.102$ & $0.297 \pm 0.025$ & 0 & $0.065 \pm 0.002$ \\
\hline 53 & $\beta$-Ionone & 37.39 & $0.554 \pm 0.048$ & $0.179 \pm 0.015$ & $0.922 \pm 0.075$ & $2.333 \pm 0.057$ \\
\hline 54 & Pentadecane & 37.62 & 0 & $0.523 \pm 0.043$ & $0.439 \pm 0.036$ & $1.786 \pm 0.044$ \\
\hline 55 & N-(1H-1,3-Benzimidazol-2-ylmethyl)-4-methoxy-benzamide & 38.31 & $1.646 \pm 0.143$ & $0.047 \pm 0.004$ & $0.629 \pm 0.051$ & $0.475 \pm 0.012$ \\
\hline 56 & Malonic acid & 38.50 & $\overline{0}$ & $\overline{0}$ & $1.177 \pm 0.096$ & $3.118 \pm 0.076$ \\
\hline 57 & $\beta$-Bisabolene & 38.81 & 0 & $0.158 \pm 0.013$ & $0.119 \pm 0.010$ & $0.54 \pm 0.013$ \\
\hline 58 & cis-Sesquisabinene & 38.87 & $2.302 \pm 0.200$ & $0.640 \pm 0.053$ & $2.530 \pm 0.0205$ & $0.527 \pm 0.013$ \\
\hline 59 & N-Phenyl-(3-methyl-2-oxiranyl) methyl carbamate & 39.03 & $\overline{0}$ & $0.362 \pm 0.030$ & 0 & $0.287 \pm 0.007$ \\
\hline 60 & Bornyl isovalerate & 39.20 & $1.110 \pm 0.096$ & $0.143 \pm 0.012$ & $0.674 \pm 0.055$ & $0.216 \pm 0.005$ \\
\hline 61 & exo-3-Methyl-1,7,7-trimethylbicyclo[2,2,1]hept-2-yl butanoate & 39.50 & 0 & $0.143 \pm 0.012$ & $\overline{0}$ & $2.464 \pm 0.060$ \\
\hline 62 & 2.6-Dimethylnon-1-en-3-yn-5-yl valeric acid & 39.81 & $0.708 \pm 0.061$ & $\overline{0}$ & 0 & $1.249 \pm 0.031$ \\
\hline 63 & cis- $\alpha$-Bisabolene & 40.33 & $0.621 \pm 0.054$ & 0 & $0.533 \pm 0.043$ & $0.187 \pm 0.005$ \\
\hline 64 & trans-Sesquisabinene & 40.50 & 0 & 0 & $0.467 \pm 0.038$ & $0.213 \pm 0.005$ \\
\hline 65 & 1-Phenylthio-3-(1-piperidyl)-propan-2ol & 40.61 & $0.894 \pm 0.078$ & 0 & $\overline{0}$ & $0.134 \pm 0.003$ \\
\hline 66 & 6-epi-Shyobunol & 40.79 & 0 & $0.139 \pm 0.011$ & 0 & $2.376 \pm 0.058$ \\
\hline 67 & Photocitral B & 40.97 & $0.779 \pm 0.068$ & $0.356 \pm 0.029$ & $2.119 \pm 0.0172$ & $0.282 \pm 0.007$ \\
\hline 68 & $N$-(4-Methoxyphenyl)-propanamide & 41.42 & $1.247 \pm 0.019$ & $0.339 \pm 0.028$ & $1.212 \pm 0.098$ & $2.537 \pm 0.062$ \\
\hline 69 & Menthyl acetate & 41.68 & 0 & $0.254 \pm 0.021$ & $0.901 \pm 0.073$ & $0.08 \pm 0.002$ \\
\hline 70 & (-)-Isolongifolol methyl ether & 42.09 & 0 & 0 & 0 & $0.207 \pm 0.005$ \\
\hline
\end{tabular}


Table 1. Cont

\begin{tabular}{|c|c|c|c|c|c|c|}
\hline \multirow{2}{*}{ No. } & \multirow{2}{*}{ Volatile Compounds } & \multirow{2}{*}{$\mathbf{R I}^{*}$} & \multicolumn{4}{|c|}{ Relative Peak Area (\%) } \\
\hline & & & Flower & Stem & Leaf & Root \\
\hline 71 & 5,5-Dimethyl-4-(3-methyl-1,3-butadienyl)-1-oxaspiro[2,5]octane & 42.20 & $1.121 \pm 0.097$ & 0 & 0 & $0.127 \pm 0.003$ \\
\hline 72 & 3,3-dichlorodihydro-2 $(3 H)$-furanone & 42.38 & $\overline{0}$ & 0 & 0 & $0.313 \pm 0.008$ \\
\hline 73 & Z-9-Pentadecenol & 42.74 & $2.454 \pm 0.213$ & $1.530 \pm 0.126$ & $3.563 \pm 0.289$ & $0.544 \pm 0.013$ \\
\hline 74 & Acetonylacetone dioxime & 42.97 & 0 & $0.122 \pm 0.010$ & 0 & $\overline{0}$ \\
\hline 75 & 1-Adamantylmethyl 3-methyl-2-butenoate & 43.27 & $1.593 \pm 0.138$ & $1.392 \pm 0.115$ & $0.223 \pm 0.018$ & $0.221 \pm 0.005$ \\
\hline 76 & 3-(Methylthio)phenyl isothiocyanate & 43.89 & 0 & $0.527 \pm 0.044$ & $0.921 \pm 0.075$ & $0.041 \pm 0.001$ \\
\hline 77 & 1-Adamantylmethyl octanoic acid & 44.20 & $0.780 \pm 0.068$ & $11.040 \pm 0.912$ & $\overline{0}$ & $0.123 \pm 0.003$ \\
\hline 78 & 2-(Methylthio)-benzothialzole & 44.43 & $1.013 \pm 0.088$ & $0.300 \pm 0.025$ & $0.280 \pm 0.023$ & $0.06 \pm 0.001$ \\
\hline 79 & 3-Methyl-2(3H)-benzothiazolethione & 44.74 & $0.596 \pm 0.052$ & $0.439 \pm 0.036$ & $0.654 \pm 0.053$ & $0.046 \pm 0.001$ \\
\hline 80 & Pentanoic acid & 45.25 & $1.072 \pm 0.09$ & $0.531 \pm 0.044$ & $0.522 \pm 0.042$ & $0.165 \pm 0.004$ \\
\hline 81 & Terpinyl acetate & 45.42 & $1.550 \pm 0.135$ & $3.000 \pm 0.248$ & 0 & 0 \\
\hline 82 & $p$-Methoxybenzylazidoformate & 46.29 & $0.975 \pm 0.085$ & $2.492 \pm 0.206$ & $1.051 \pm 0.085$ & $0.439 \pm 0.011$ \\
\hline 83 & 5-Amino-ethyl ester $[1,2,4]$ triazolo[4.3-a]pyrimidine-6-carboxylate & 46.47 & $0.611 \pm 0.053$ & $0.288 \pm 0.024$ & 0 & 0 \\
\hline 84 & 1,3,-Trimethyl-2-hydroxymethyl-3,3-dimethyl-4(3-methylbut-2-eny)-cyclohexene & 46.71 & $1.044 \pm 0.091$ & $0.766 \pm 0.063$ & 0 & 0 \\
\hline 85 & 5-Butyl-6-hexyloctahydro-1H-indene & 47.14 & $0.992 \pm 0.086$ & $0.229 \pm 0.019$ & 0 & 0 \\
\hline 86 & 4-Hydroxy-2-hydroxymethyl-6-methylpyrimidine & 47.47 & $0.456 \pm 0.040$ & $\overline{0}$ & 0 & 0 \\
\hline 87 & Murolan-3,9(11)-diene-10-peroxy & 47.76 & $0.888 \pm 0.077$ & $0.355 \pm 0.029$ & $0.482 \pm 0.039$ & $0.131 \pm 0.003$ \\
\hline 88 & Methanone & 48.04 & $0.414 \pm 0.036$ & 0.2750 .023 & $\overline{0}$ & $\overline{0}$ \\
\hline 89 & 8-Chlorooctyl isobutyl carbonate & 48.29 & $0.522 \pm 0.045$ & $0.134 \pm 0.011$ & 0 & 0 \\
\hline 90 & 1,2-Pentanediol & 48.72 & $0.719 \pm 0.062$ & 0 & 0 & $0.031 \pm 0.001$ \\
\hline 91 & Bicyclogermacrene & 49.21 & $1.642 \pm 0.143$ & 0 & 0 & 0 \\
\hline 92 & Larixone & 49.39 & $\overline{0}$ & $0.159 \pm 0.013$ & 0 & 0 \\
\hline 93 & Ursane-3,16-diol & 49.70 & $0.856 \pm 0.074$ & $0.921 \pm 0.076$ & $0.640 \pm 0.052$ & $0.04 \pm 0.001$ \\
\hline 94 & Dodecahydro-3,8,8,11a-tetramethyl-5H-3,5a-epoxynaphth[2,1-c]oxepin & 49.94 & $1.248 \pm 0.108$ & $0.354 \pm 0.029$ & 0 & 0 \\
\hline 95 & Hexahydro-5-methyl-1-phenyl-1,3,5-triazine-2-thione & 50.27 & $\overline{0}$ & $1.515 \pm 0.125$ & $0.240 \pm 0.019$ & 0 \\
\hline 96 & $(2 R, 4 R)-p$-Mentha-6,8-diene-2-hydroperoxide & 50.71 & $0.793 \pm 0.069$ & $0.271 \pm 0.022$ & 0 & 0 \\
\hline 97 & 4-(Diethoxyphosphiniyl)butanoic acid & 51.18 & $0.259 \pm 0.022$ & $0.246 \pm 0.020$ & 0 & 0 \\
\hline 98 & 2,2-Dimethylpropanoic acid & 51.74 & $0.878 \pm 0.076$ & $0.076 \pm 0.006$ & 0 & 0 \\
\hline 99 & Nerolidol isobutyrate & 52.17 & $0.587 \pm 0.051$ & $0.253 \pm 0.021$ & 0 & 0 \\
\hline 100 & Longifolenaldehyde & 52.31 & $0.510 \pm 0.044$ & 0 & 0 & 0 \\
\hline 101 & Caryophyllene oxide & 52.67 & $0.359 \pm 0.031$ & $17.454 \pm 1.442$ & 0 & 0 \\
\hline 102 & Carbamic acid & 52.89 & $0.670 \pm 0.058$ & $0.939 \pm 0.078$ & $0.220 \pm 0.018$ & $0.061 \pm 0.001$ \\
\hline 103 & Ptenin-6-carboxylic acid & 53.22 & $1.473 \pm 0.127$ & $2.224 \pm 0.183$ & $\overline{0}$ & $0.024 \pm 0.001$ \\
\hline 104 & 2,4,4-Trimethyl-3-hydroxymethyl-5a-(3-methyl-but-2-enyl)-cyclohexene & 53.48 & $0.218 \pm 0.019$ & $0.324 \pm 0.027$ & 0 & 0 \\
\hline 105 & 2-(2-Dodecen-1yl)succinic acid & 53.76 & $0.801 \pm 0.070$ & $0.145 \pm 0.012$ & 0 & 0 \\
\hline
\end{tabular}


Table 1. Cont

\begin{tabular}{|c|c|c|c|c|c|c|}
\hline \multirow{2}{*}{ No. } & \multirow{2}{*}{ Volatile Compounds } & \multirow{2}{*}{$\mathbf{R I}$ * } & \multicolumn{4}{|c|}{ Relative Peak Area (\%) } \\
\hline & & & Flower & Stem & Leaf & Root \\
\hline 106 & Ginsenol & 54.37 & $0.294 \pm 0.026$ & $0.394 \pm 0.033$ & 0 & 0 \\
\hline 107 & Costunolide & 54.55 & $\overline{0}$ & $0.114 \pm 0.009$ & 0 & 0 \\
\hline 108 & 1-Formyl-2,2,6-trimethyl-3-(3-methyl-but-2-enyl)-6-cyclohexene & 54.95 & $3.140 \pm 0.273$ & $1.125 \pm 0.093$ & 0 & 0 \\
\hline 109 & 4-epi-Cubedol & 55.61 & $0.819 \pm 0.071$ & $0.313 \pm 0.026$ & 0 & 0 \\
\hline 110 & 5-(6-Bromodecahydro-2-hydroxy-2,5,5a,8a-tetramethyl-1-naphthalene)-1,2-pentanediol & 55.82 & $0.441 \pm 0.038$ & 0 & 0 & 0 \\
\hline 111 & $(8 \mathrm{~S}, 14)$-Cedran-diol & 56.00 & $0.855 \pm 0.074$ & $0.914 \pm 0.075$ & 0 & 0 \\
\hline 112 & Cedrol & 56.39 & $0.379 \pm 0.033$ & 0 & 0 & 0 \\
\hline 113 & 8-Propoxy cedrane & 56.65 & $0.187 \pm 0.016$ & $0.078 \pm 0.006$ & 0 & 0 \\
\hline 114 & 5,6,6-Trimethyl-undeca-3,4-diene-2,10-dione & 56.81 & $0.311 \pm 0.027$ & $0.130 \pm 0.011$ & 0 & 0 \\
\hline 115 & Octahydro $[1,2]$ azaborino $[1,2-a][1,2]$ azaborine & 57.53 & $0.535 \pm 0.046$ & 0 & 0 & 0 \\
\hline 116 & 2.6-Dimethylnon-1-en-3-yn-5-yl valeric acid & 58.56 & $0.292 \pm 0.025$ & 0 & 0 & 0 \\
\hline 117 & Terephthalic acid & 58.80 & $0.174 \pm 0.015$ & $7.487 \pm 0.618$ & 0 & 0 \\
\hline 118 & Adamantane & 58.98 & $0.457 \pm 0.040$ & $0.944 \pm 0.078$ & 0 & 0 \\
\hline 119 & 4,7-Methano-3,6,8-methenocyclopent[a]indene & 59.80 & $0.105 \pm 0.009$ & 0 & 0 & 0 \\
\hline 120 & 4-Dimethylamino-2-methyl-1-phenyl-butan-2-ol & 60.11 & $0.316 \pm 0.027$ & $0.376 \pm 0.031$ & 0 & 0 \\
\hline 121 & $( \pm)$ cis-3,4-Dimethyl-2-phenyltetrahydro-1,4-thiazine & 61.09 & $0.185 \pm 0.016$ & $\overline{0}$ & 0 & 0 \\
\hline 122 & 2-Isopropyl-6-phenylnicotinonitrile & 62.82 & $0.251 \pm 0.022$ & 0 & 0 & 0 \\
\hline 123 & 2-Propenoic acid & 64.22 & $0.242 \pm 0.021$ & $0.300 \pm 0.025$ & 0 & 0 \\
\hline 124 & Hexanedioic acid & 64.57 & $0.476 \pm 0.041$ & $7.976 \pm 0.659$ & 0 & 0 \\
\hline 125 & Methadone $N$-oxide & 65.01 & $0.115 \pm 0.010$ & $\overline{0}$ & 0 & 0 \\
\hline 126 & Isophthalic acid & 67.21 & $0.591 \pm 0.051$ & $1.164 \pm 0.096$ & 0 & 0 \\
\hline 127 & Diisooctyl phthalate & 68.01 & $0.276 \pm 0.024$ & $0.420 \pm 0.035$ & 0 & 0 \\
\hline \multirow[t]{2}{*}{128} & Di-(2-methyoxyethyl) Isophthalate & 69.18 & $\overline{0}$ & $0.113 \pm 0.009$ & 0 & 0 \\
\hline & Total & & $65.33 \pm 5.564$ & $84.057 \pm 6.932$ & $74.916 \pm 6.073$ & $95.661 \pm 2.342$ \\
\hline
\end{tabular}

${ }^{*}$ Retent ion time (min). 


\subsection{Amounts of Valerenic Acid and Its Derivatives}

Valerenic acid and its derivatives, acetoxyvalerenic acid and hydroxyvalerenic acid, were extracted from different organs (flower, stem, leaf, and root) of $V$. fauriei and analyzed with HPLC. Valerenic acid and its derivatives were detected only in roots (Table 2). However, flowers, stems, and leaves have not shown detectable levels of the three compounds. The root extract had an average of $69.450 \mu \mathrm{g}$ of valerenic acid per $\mathrm{g}$ of dry weight. Acetoxyvalerenic acid (32.234 $\mathrm{gg} / \mathrm{g}$ dry weight) also had a high accumulation in the root. However, hydroxyvalerenic acid was not detected in any organ.

Table 2. Amounts of valerenic acid and its derivatives in $V$. fauriei roots.

\begin{tabular}{cc}
\cline { 2 - 2 } Compound & Dry Weight $(\mu \mathrm{g} / \mathrm{g})$ \\
\cline { 2 - 3 } valerenic acid & $69.450 \pm 0.263$ \\
acetoxyvalerenic acid & $32.234 \pm 0.961$ \\
hydroxyvalerenic acid & n.d. ${ }^{1}$ \\
\cline { 2 - 3 } Numbers indicate the mean of three replicates \pm standard deviation. ${ }^{1}$ n.d. & $=$ not detected.
\end{tabular}

\section{Discussion}

There is considerable interest in chemical composition variation in Valeriana owing to the numerous beneficial properties attributed to consumption of the genus. Previously, the genes involved in volatile terpenoid biosynthesis had not been investigated in different organs of $V$. fauriei. Our study documents the compositional diversity and amount of volatile compounds, including valerenic acid and its derivatives, and quantifies the expression of genes involved in terpenoid biosynthetic pathways, in different organs of $V$. fauriei.

Roots had high amounts of volatile compounds but low transcript levels. Evidently, expression levels of terpenoid biosynthetic genes did not correspond with the storage of volatile constituents, indicating that products synthesized in the stem or other organs may be translocated into the root. Earlier experimental studies have found similar results. Plants often transport natural products from a synthesis site to an accumulation site [21]. For example, compounds were synthesized in the stem and leaf, then transported to the root of Astragalus membranaceus Moench [22]. In addition, Lykkesfeldt and Moller [23] have reported the synthesis and translocation of glucosinolates from leaves to seeds during development in Tropaeolum majus L. Moreover, nicotine and caffeine, which are primarily produced roots, are transferred to leaves in Arabidopsis thaliana (L.) Heynh. [24]. Translocation of secondary metabolites from source cells to neighboring cells, or even further to other tissues usually occurs in plants. Volatile terpenes are emitted by the transcript levels of biosynthetic enzymes, and transporter for their emission is not required. However, the strong evidence which demonstrates to deny the presence of broad-specificity transporters for the emission of volatiles has not yet been identified. In addition, the mechanism for the long-distance translocation of volatiles has not been investigated in plants [25]. Therefore, we should carry out more experiments such as feeding of radio labels to $V$. fauriei tissues to clarify the transport and accumulation of volatile compounds in further study.

Various taxa of Valeriana, including V. officinalis L., V. jatamansi Jones, V. officinalis var. latifolia Miq., $V$. amurensis P. Smirn. ex Kom., V. fauriei, and V. alternifolia Bunge var. stolonifera A.I. Baranov \& Skvortsov, have been the focus of scientific studies [18,26,27]. Several studies have included experiments to isolate and identify chemical constituents including sesquiterpenoids and iridoid glycosides from $V$. fauriei [26,27]. In our study, we identified 128 volatile compounds in $V$. fauriei by GC/MS. Although most volatile compounds had high concentrations in the roots, each organ had several distinctive compounds that served as biochemical markers. For example, isovalerenic acid, which causes the malodorous characteristic, was accumulated solely in the root of $V$. fauriei. In addition, we found that borneol and bornyl acetate were the most abundant monoterpenes in the roots of $V$. fauriei, in agreement with the results from previous studies. Wang et al. [18] documented that $V$. officinalis var. latifolia contained the highest amounts of bornyl acetate $(23.93 \%)$ in the roots. To date, 
more than 100 members of TPSs have been identified from various plant species [13]. The action of these enzymatic family lead to structurally diverse cyclic and acyclic monoterpenes and sesquiterpenes [28]. Specific monoterpene synthase catalyze from GPP to various skeletons which are the precursors such as olefins, alcohols and diphosphate esters for the biosynthesis of monoterpenes [29]. Also, the enzymatic cyclization in several plants including Salvia, Mentha, Tanacetum, Foeniculum, Pinus, and Citrus species has been reported [30]. Cyclization of linalyl diphosphate derived from GPP occurs to form the $\alpha$-terpinyl cation, which is the universal monocyclic intermediate. Then, bornane family such as borneol, bornyl acetate, and camphor is converted by hydrolysis, oxidation, and rearrangement of carbocylic skeleton [31]. It has been investigated that bornyl diphosphate synthase acts on production of bornane-type monoterpenes in higher plants [32]. The characteristic compound valerenic acid, along with its derivatives and iridoids, are primarily accumulated in the roots and rhizomes of Valeriana species [20].Similarly, we found that high amounts of valerenic acid and hydroxyvalerenic acid were detected only in the roots of $V$. fauriei. Navarrete et al. [33] performed quantitative analyses of valerenic acids (valerenic, hydroxyvalerenic, and acetoxyvalerenic) in Valeriana species including $V$. officinalis, $V$. edulis Nutt. ex Torr. \& A. Gray, V. sitchensis Bong., and V. jatamansi, but only $V$. officinalis and $V$. sitchensis accumulated these compounds.

In summary, we analyzed organ specificity in general-common MVA and MEP biosynthesis by measuring transcript levels of related biosynthetic genes. We also measured the accumulation of volatile compounds, and the presence of valerenic acid and its derivatives, in specific organs. We separated and identified 128 volatile compounds in $V$. fauriei cultivated in South Korea. Volatile compounds were detected in all tested organs, but most of the volatile compounds were stored in the root. In contrast, high levels of gene expression predominantly occurred in the stem. Therefore, terpenoids appear to be translocated after biosynthesis and are stored in specific organs.

\section{Materials and Methods}

\subsection{Plant Materials and Growth Conditions}

V. fauriei plants were grown at the experimental station, National Institute of Horticultural and Herbal Science (NIHHS), Rural Development Administration (RDA), Pyeongchang, Gangwon-do, South Korea. The plants were grown outdoors in a field from 2013 to 2014. Leaves, stems, flowers, and roots were collected in July 2014 (Figure 4) and then immediately frozen in liquid nitrogen. Different organs from three individual plants were harvested under the same experimental settings. The materials were stored at $-80{ }^{\circ} \mathrm{C}$ until they were used.
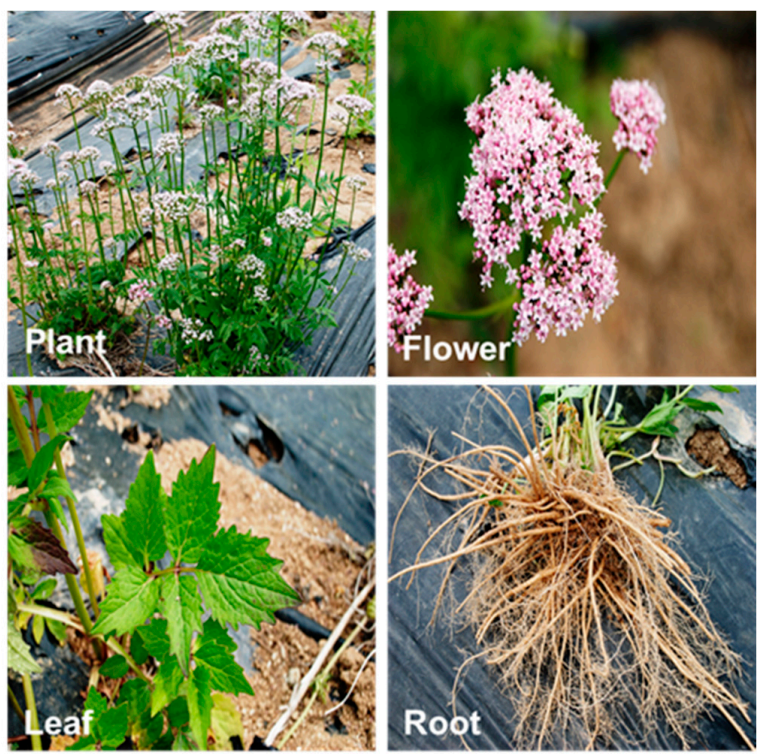

Figure 4. Photographs of two-year-old V. fauriei grown in Pyeongchang, Gangwon-do, South Korea. 


\subsection{Total RNA Extraction and cDNA Preparation}

Total RNA was isolated from each organ using a Total RNA Mini Kit (Geneaid, New Taipei City, Taiwan) following the manufacturer's instructions using three biological replicates. DNase I (Qiagen, Hilden, North Rhine-Westphalia, Germany) was used to remove genomic DNA. RNA concentration and purity were confirmed with agarose gel electrophoresis and a NanoVue Plus Spectrophotometer (GE Healthcare Bio-Science Corp., Piscataway, NJ, USA). cDNA was synthesized from $1 \mu \mathrm{g}$ of total RNA using the ReverTra Ace- $\alpha$ Kit (Toyobo, Osaka, Japan).

\section{3. qRT-PCR Analysis}

All gene specific primers used were designed as previously described by Park et al [34] (Table A1). qRT-PCR was performed with a BIO-RAD CFX96 Real-time PCR system (Bio-Rad Laboratories, Hercules, CA, USA) with the 2X Real-Time PCR smart mix (Solgent Co., Ltd. Daejeon, Korea) under the following conditions: initial denaturation at $95^{\circ} \mathrm{C}$ for $15 \mathrm{~min}, 39$ cycles of denaturation for $20 \mathrm{~s}$ at $95{ }^{\circ} \mathrm{C}$ and annealing for $20 \mathrm{~s}$ at $72{ }^{\circ} \mathrm{C}$, with a final extension at $72{ }^{\circ} \mathrm{C}$ for $10 \mathrm{~min}$. Melting curves were analyzed to verify reaction specificity. Means and standard deviations were calculated from three independent biological replicates for each sample and compared by organ.

\subsection{GC and GC-MS}

Samples (10 g) of the fresh material (leaves, stems, flowers, and roots) were weighed and placed in vials with $25 \mathrm{~mL}$ of headspace. A fused-silica fiber covered with a 75- $\mu \mathrm{m}$-thick layer of carboxen/polydimethylsiloxane (CAR/PDMS) was used to absorb volatile compounds. It was exposed in the headspace in the vials at $25^{\circ} \mathrm{C}$ for $20 \mathrm{~min}$, then removed from vials and introduced directly into the GC injector where thermal desorption of the analysis was performed at $250{ }^{\circ} \mathrm{C}$ for $3 \mathrm{~min}$.

GC analysis was carried out with an Agilent 6890N GC mainframe fitted with an HP-5 fused-silica capillary column ( $30 \mathrm{~m} \times 0.32 \mathrm{~mm}$ i.d., $0.25 \mu \mathrm{m}$ film thickness; Agilent, Santa Clara, CA, USA) and a flame ionization detector (FID). The injector and detector temperatures for each analysis were $250{ }^{\circ} \mathrm{C}$ and $280^{\circ} \mathrm{C}$, respectively. The carrier gas was nitrogen, with a flow rate of $1.0 \mathrm{~mL} / \mathrm{min}$. The column temperature was maintained at $50^{\circ} \mathrm{C}$ for $5 \mathrm{~min}$ and then programmed as follows: increase from $50^{\circ} \mathrm{C}$ to $260^{\circ} \mathrm{C}$ at a rate of $3^{\circ} \mathrm{C} \cdot \mathrm{min}^{-1}$, increase from $260{ }^{\circ} \mathrm{C}$ to $280^{\circ} \mathrm{C}$ at a rate of $10^{\circ} \mathrm{C} \cdot \mathrm{min}^{-1}$, and hold at $280^{\circ} \mathrm{C}$ for $5 \mathrm{~min}$.

GC-MS analysis was performed on a Polaris Q GC/MS (Thermo Finnigan, Waltham, MA, USA) coupled with an HP-5 fused-silica capillary column $(30 \mathrm{~m} \times 0.32 \mathrm{~mm}$ i.d., film thickness $0.25 \mu \mathrm{m}$; Agilent). Helium was used as the carrier gas at a flow rate of $1.0 \mathrm{~mL} / \mathrm{min}$. The mass spectra were obtained with an ionization voltage of $70 \mathrm{eV}$, trap current of $250 \mu \mathrm{A}$, and ion source temperature of $200{ }^{\circ} \mathrm{C}$. The oven temperature program was the same as described previously, and injections were made in splitless mode. Volatile compounds were separated and identified based on comparison of the mass spectra with the National Institute of Standards and Technology (NIST) atomic spectra database, Wiley Registry of Mass Spectral Data, and the related literature. Total ion current chromatograms were recorded in a mass range of $40-400 \mathrm{amu}$. The analyses were performed using three biological replicates.

\subsection{Measurement of Valerenic Acid and Derivatives}

All samples were lyophilized at $-80^{\circ} \mathrm{C}$ for $72 \mathrm{~h}$. Dried samples were ground into a fine powder using a mortar and pestle. One g of powdered sample was extracted with $10 \mathrm{~mL}$ of $90 \%(v / v)$ methanol at room temperature for $30 \mathrm{~min}$ and then centrifuged at $19354 \mathrm{rcf}$ for $10 \mathrm{~min}$. The supernatant was transferred to a new tube. Centrifugation and supernatant transfer were repeated two more times. The final extract was adjusted to $1 \mathrm{~mL}$ through evaporation using a LABOROTA 4000 rotary evaporator (Heidolph Instruments $\mathrm{GmbH}$, Schwabach, Bavaria, Germany). The solution was filtered with a $0.45 \mu \mathrm{m}$ Acrodisc syringe filter (Pall Corp.; Port Washington, NY, USA) for HPLC analysis. 
HPLC analysis was performed with a Futecs model NS-4000 HPLC apparatus (Daejeon, Korea) and a C18 column ( $\mu$ Bondapak ${ }^{\mathrm{TM}}$ C18 $10 \mu \mathrm{m} 125 \AA 3.9 \times 300$ nm column, Waters, Milford, MA, USA). The mobile phase had a gradient prepared from mixtures of acetonitrile and $0.25 \%$ phosphoric acid, and the column temperature was maintained at $30^{\circ} \mathrm{C}$. The flow rate was maintained at $0.7 \mathrm{~mL} / \mathrm{min}$. Twenty microliters of the solution was injected into the HPLC, and the detection wavelength was set at $221 \mathrm{~nm}$. The analysis was performed using three biological replicates.

\subsection{Statistical Analysis}

The data for gene expression and valerenic acid and its derivatives contents were analyzed by Microsoft EXCEL (Version. 2010, Microsoft Corporation, Seattle, WA, USA). Statistical analyses for the relative peak areas were conducted using XL-STAT, version 2013 (Addinsoft, NY, USA).

Acknowledgments: This work was carried out with the support of "National Institute of Horticultural and Herbal Science (NIHHS)" Rural Development Administration (RDA), Korea. The authors extend their sincere appreciation to the Deanship of Scientific Research at King Saud University for its funding through the Prolific Research Group (PRG-1437-28).

Author Contributions: N.A.A-D. and S.U.P. conceived and designed the experiments. Y.J.P., S.S.L., Y.B.K., M.V.A. and S.W.L. performed the experiments and wrote the paper.

Conflicts of Interest: The authors declare no conflict of interest.

\section{Abbreviations}

The following abbreviations are used in this manuscript:

$\begin{array}{ll}\text { AACT } & \text { Acetocetyl-CoA thiolase } \\ \text { CAR/PDMS } & \text { carboxen/polydimethylsiloxane } \\ \text { CMK } & \text { 4-(cytidine 5'-diphosphate)-2-C-methyl-D-erythritol kinase } \\ \text { DMAPP } & \text { dimethylallyl diphosphate } \\ \text { DXP } & \text { 1-deoxy-D-xylulose 5-phosphate } \\ \text { DXR } & \text { DXP reductoisomerase } \\ \text { DXS } & \text { DXP synthase } \\ \text { FDS } & \text { farndsyl diphosphate synthase } \\ \text { FPP } & \text { farnesyl diphosphate } \\ \text { GA-3P } & \text { glyceraldehyde-3-phosphae } \\ \text { GC } & \text { gas chromatography } \\ \text { GC/MS } & \text { gas chromatography-mass spectrometry } \\ \text { GDS } & \text { geranyl diphosphate synthase } \\ \text { GGPP } & \text { geranyl geranyl diphosphate } \\ \text { GPP } & \text { geranyl diphosphate } \\ \text { HDR } & \text { (E)-4-hydroxy-3-methylbut 2-enyl diphosphate reductase } \\ \text { HDS } & \text { (E)-4-hydroxy-3-methylbut-2-enyl diphosphate synthase } \\ \text { HMG-CoA } & \text { 3-hydroxy-3-methylglutaryl-CoA } \\ \text { HMGR } & \text { HMG-CoA reductase } \\ \text { HMGS } & \text { HMG-CoA synthase } \\ \text { IDI } & \text { isopentenyl diphosphate isomerase } \\ \text { IPP } & \text { isopentenyl diphosphate } \\ \text { MCT } & \text { 2-C-methyl-D-erythritol 4-phosphate cytidylyltransferase } \\ \text { MDS } & \text { 2-C-methyl-D-erythritol 2,4-cyclodiphosphate synthase } \\ \text { MEP } & \text { 2-C-methyl-D-erythritol 4-phosphate } \\ \text { MK } & \text { mevalonate kinase } \\ \text { MVA } & \text { mevalonae } \\ \text { MVD } & \text { mevalonate diphosphate decarboxylase } \\ \text { qRT-PCR } & \text { quantitative Real-time PCR } \\ \text { PMK } & \text { phosphomevalonate kinase } \\ \text { TPS } & \text { terpene synthase } \\ & \end{array}$




\section{Appendix}

Table A1. Primers sets used in the experiments and their efficiency (\%) for qRT-PCR.

\begin{tabular}{|c|c|c|c|}
\hline Primer & Sequence $\left(5^{\prime}\right.$ to $\left.3^{\prime}\right)$ & Amplication (bp) & Primer Efficiency (\%) \\
\hline VfAACT-F & ATCGGGCATGAAAGCAACCA & \multirow[b]{2}{*}{129} & \multirow[b]{2}{*}{97.9} \\
\hline VfAACT-R & GATCCСTTCCTTGCTTCCGCTA & & \\
\hline VfHMGS-F & TGGTGGAACTGCAGCATTGTTC & \multirow[b]{2}{*}{131} & \multirow[b]{2}{*}{98.8} \\
\hline VfHMGS-R & CCGCACTGTCTGTGCACACGA & & \\
\hline VfHMGR-F & TCAGATGCCCTGCCTCTTCC & \multirow[b]{2}{*}{102} & \multirow{2}{*}{100.2} \\
\hline VfHMGR-R & GATCTTCTCGCGCCACCTTG & & \\
\hline VfMK-F & CGGCGGCTGCGTATTGACT & \multirow[b]{2}{*}{133} & \multirow[b]{2}{*}{107.1} \\
\hline VfMK-R & GCAAATCTCGAGCCCATTGC & & \\
\hline VfPMK-F & GAGCCGGAATCACAGACGGA & \multirow{2}{*}{146} & \multirow{2}{*}{93.3} \\
\hline VfPMK-R & CTCCACGTCTTTGCGAGGCT & & \\
\hline VfMVD-F & AATGGAATCGCGCTGAAGGA & \multirow[b]{2}{*}{105} & \multirow[b]{2}{*}{98} \\
\hline VfMVD-R & CACAGCATTCGGCCCAGCAT & & \\
\hline VfIDI-F & AGCAGATGCAGGCGAAGAGG & \multirow{2}{*}{111} & \multirow{2}{*}{93.3} \\
\hline VfIDI-R & GCCTCGCTTAAGTTCCCGTTCT & & \\
\hline VfFDS-F & TGATGACGACGGCAAGGAGA & \multirow[b]{2}{*}{131} & \multirow[b]{2}{*}{94.9} \\
\hline VfFDS-R & CACCAACCAAGTGAGCATGCAAGA & & \\
\hline VfDXS-F & GCCCAATACCACCTGTCGGA & \multirow{2}{*}{121} & \multirow{2}{*}{95.8} \\
\hline VfDXS-R & TGCATCGGTCCACCAATCTG & & \\
\hline VfDXR-F & AGAACTCCGGTCATTGTGCCA & \multirow[b]{2}{*}{120} & \multirow[b]{2}{*}{100.1} \\
\hline VfDXR-R & CCGCCTCGATCTTTGCAAGTTA & & \\
\hline VfMCT-F & TCAGTTGCTCTGCAAATGGGAGT & \multirow{2}{*}{104} & \multirow{2}{*}{102.2} \\
\hline VfMCT-R & TCCCATTCTTGTGCCСTTTCC & & \\
\hline VfCMK-F & GCACCATTGTTGGGATCGGT & \multirow[b]{2}{*}{113} & \multirow[b]{2}{*}{82.6} \\
\hline VfCMK-R & CTCGTTCTCGGCTCGTGTGA & & \\
\hline VfMDS-F & TGCAGCTACTGCTGCTGTGGA & \multirow{2}{*}{144} & \multirow{2}{*}{70.6} \\
\hline VfMDS-R & GGTATGTTGATGCCGCCGAT & & \\
\hline VfHDS-F & CTGACAGGCGGGCACAGTTT & \multirow{2}{*}{134} & \multirow{2}{*}{97.1} \\
\hline VfHDS-R & GCCGATTCGCATAGCTCTTCC & & \\
\hline VfHDR-F & CCGAAGCAATCGGGAAGTTG & \multirow{2}{*}{112} & \multirow{2}{*}{95} \\
\hline VfHDR-R & CGCTCTTGAGTAGCGTCGCA & & \\
\hline VfGDS-F & TAGCAGTGCTGGCGGGAGAT & \multirow[b]{2}{*}{145} & \\
\hline VfGDS-R & TCGCGCGTCGTACTCATTTG & & 102.8 \\
\hline
\end{tabular}

\section{References}

1. Pichersky, E.; Noel, J.P.; Dudareva, N. Biosynthesis of plant volatiles: Nature's diversity and ingenuity. Science 2006, 311, 808-811. [CrossRef] [PubMed]

2. Knudsen, J.T.; Eriksson, R.; Gershenzon, J.; Ståhl, B. Diversity and distribution of floral scent. Bot. Rev. 2006, 72, 1-120. [CrossRef]

3. Pichersky, E.; Gershenzon, J. The formation and function of plant volatiles: Perfumes for pollinator attraction and defense. Curr. Opin. Plant Biol. 2002, 5, 237-243. [CrossRef]

4. Dudareva, N.; Pichersky, E. Metabolic engineering of plant volatiles. Curr. Opin. Biotechnol. 2008, 19, 181-189. [CrossRef] [PubMed]

5. Peñuelas, J.; Llusià, J. Plant VOC emissions: Making use of the unavoidable. Trends Ecol. Evol. 2004, 19, 402-404. [CrossRef] [PubMed]

6. Goff, S.A.; Klee, H.J. Plant volatile compounds: Sensory cues for health and nutritional value? Science 2006, 311, 815-819. [CrossRef] [PubMed]

7. Maffei, M.E.; Gertsch, J.; Appendino, G. Plant volatiles: Production, function and pharmacology. Nat. Prod. Rep. 2011, 28, 1359-1380. [CrossRef] [PubMed]

8. Dicke, M.; Loreto, F. Induced plant volatiles: From genes to climate change. Trends Plant Sci. 2010, 15, 115-117. [CrossRef] [PubMed] 
9. Tholl, D. Terpene synthases and the regulation, diversity and biological roles of terpene metabolism. Curr. Opin. Plant Biol. 2006, 9, 297-304. [CrossRef] [PubMed]

10. Dudareva, N.; Pichersky, E.; Gershenzon, J. Biochemistry of plant volatiles. Plant Physiol. 2004, 135, 1893-1902. [CrossRef] [PubMed]

11. Koyama, T.; Ogura, K. Isopentenyl diphosphate isomerase and prenyltransferases. Compr. Nat. Prod. Chem. 1999, 2, 69-96.

12. Liang, P.H.; Ko, T.P.; Wang, A.H.J. Structure, mechanism and function of prenyltransferases. Eur. J. Biochem. 2002, 269, 3339-3354. [CrossRef] [PubMed]

13. Dudareva, N.; Klempien, A.; Muhlemann, J.K.; Kaplan, I. Biosynthesis, function and metabolic engineering of plant volatile organic compounds. New Phytol. 2013, 198, 16-32. [CrossRef] [PubMed]

14. Besser, K.; Harper, A.; Welsby, N.; Schauvinhold, I.; Slocombe, S.; Li, Y.; Dixon, R.A.; Broun, P. Divergent regulation of terpenoid metabolism in the trichomes of wild and cultivated tomato species. Plant Physiol. 2009, 149, 499-514. [CrossRef] [PubMed]

15. Mathela, C.S.; Chanotiya, C.S.; Sammal, S.S.; Pant, A.K.; Pandey, S. Compositional diversity of terpenoids in the himalayan valeriana genera. Chem. Biodivers. 2005, 2, 1174-1182. [CrossRef] [PubMed]

16. Wang, Y.; Jin, L.; Yu, S.; Shi, Q.; Gu, Y.; Kiyota, H. Chemical constituents of plants from the genus valeriana. Mini Rev. Org. Chem. 2010, 7, 161-172. [CrossRef]

17. Houghton, P.J. The scientific basis for the reputed activity of valerian. J. Pharm. Pharmacol. 1999, 51, 505-512. [CrossRef] [PubMed]

18. Chen, H.-W.; Wei, B.-J.; He, X.-H.; Liu, Y.; Wang, J. Chemical components and cardiovascular activities of Valeriana spp. Evid. Based Complement. Altern. Med. 2015, 2015. [CrossRef]

19. Bos, R.; Woerdenbag, H.J.; Van Putten, F.; Hendriks, H.; Scheffer, J. Seasonal variation of the essential oil, valerenic acid and derivatives, and velopotriates in valeriana officinalis roots and rhizomes, and the selection of plants suitable for phytomedicines. Planta Med. 1998, 64, 143-147. [CrossRef] [PubMed]

20. Oprean, R.; Oprean, L.; Tamas, M.; Sandulescu, R.; Roman, L. Essential oils analysis. II. Mass spectra identification of terpene and phenylpropane derivatives. J. Pharm. Biomed. Anal. 2001, 24, 1163-1168. [CrossRef]

21. Dhaubhadel, S.; McGarvey, B.D.; Williams, R.; Gijzen, M. Isoflavonoid biosynthesis and accumulation in developing soybean seeds. Plant Mol. Biol. 2003, 53, 733-743. [CrossRef] [PubMed]

22. Kim, Y.B.; Thwe, A.A.; Li, X.; Tuan, P.A.; Lee, S.; Lee, J.W.; Arasu, M.V.; Al-Dhabi, N.A.; Park, S.U. Accumulation of astragalosides and related gene expression in different organs of astragalus membranaceus BGE. Var mongholicus (BGE.). Molecules 2014, 19, 10922-10935. [CrossRef] [PubMed]

23. Lykkesfeldt, J.; Moller, B. Synthesis of benzylglucosinolate in Tropaeolum majus L. (isothiocyanates as potent enzyme inhibitors). Plant Physiol. 1993, 102, 609-613. [PubMed]

24. Gillissen, B.; Bürkle, L.; André, B.; Kühn, C.; Rentsch, D.; Brandl, B.; Frommer, W.B. A new family of high-affinity transporters for adenine, cytosine, and purine derivatives in arabidopsis. Plant Cell 2000, 12, 291-300. [CrossRef] [PubMed]

25. Yazaki, K. Transporters of secondary metabolites. Curr. Opin. Plant Biol. 2005, 8, 301-307. [PubMed]

26. Nishiya, K.; Kimura, T.; Takeya, K.; Itokawa, H. Sesquiterpenoids and iridoid glycosides from valeriana fauriei. Phytochemistry 1994, 36, 1547-1548. [CrossRef]

27. Zhang, Z.-X.; Dou, D.-Q.; Liu, K.; Yao, X.-S. Studies on the chemical constituents of valeriana fauriei briq. J. Asian Nat. Prod. Res. 2006, 8, 397-400. [CrossRef] [PubMed]

28. Dudareva, N.; Negre, F.; Nagegowda, D.A.; Orlova, I. Plant volatiles: Recent advances and future perspectives. Crit. Rev. Plant Sci. 2006, 25, 417-440. [CrossRef]

29. Nagegowda, D.A. Plant volatile terpenoid metabolism: Biosynthetic genes, transcriptional regulation and subcellular compartmentation. Fed. Eur. Biochem. Soc. 2010, 584, 2965-2973. [CrossRef] [PubMed]

30. Croteau, R. Biosynthesis and catabolism of monoterpenoids. Chem. Rev. 1987, 87, 929-954. [CrossRef]

31. Davis, E.M.; Croteau, R. Cyclization enzymes in the biosynthesis of monoterpenes, sesquiterpenes, and diterpenes. In Biosynthesis; Springer: Berlin, Germany, 2000; pp. 53-95.

32. Adam, K.-P.; Croteau, R. Monoterpene biosynthesis in the liverwort conocephalum conicum: Demonstration of sabinene synthase and bornyl diphosphate synthase in honour of Professor G.H. Neil towers 75th birthday. Phytochemistry 1998, 49, 475-480. [CrossRef] 
33. Navarrete, A.; Avula, B.; Choi, Y.-W.; Khan, I.A. Chemical fingerprinting of valeriana species: Simultaneous determination of valerenic acids, flavonoids, and phenylpropanoids using liquid chromatography with ultraviolet detection. J. AOAC Int. 2006, 89, 8-15. [PubMed]

34. Park, Y.J.; Li, X.; Noh, S.J.; Kim, J.K.; Lim, S.S.; Park, N.I.; Kim, S.; Kim, Y.B.; Kim, Y.O.; Lee, S.W. Transcriptome and metabolome analysis in shoot and root of valeriana fauriei. BMC Genom. 2016, 17. [CrossRef] [PubMed]

Sample Availability: Not Available.

(C) 2016 by the authors; licensee MDPI, Basel, Switzerland. This article is an open access article distributed under the terms and conditions of the Creative Commons Attribution (CC-BY) license (http://creativecommons.org/licenses/by/4.0/). 